\title{
Fast-response, high-stability, and high-efficiency full-color quantum dot light-emitting diodes with charge storage layer
}

\author{
Yangbin $\mathrm{Zhu}^{1,2 \dagger}$, Yang $\mathrm{Liu}^{1 \dagger}$, Hailong $\mathrm{Hu}^{1}$, Zhongwei $\mathrm{Xu}^{1}$, Jieyu Bai ${ }^{1}$, Kaiyu Yang ${ }^{1}$, Tailiang Guo ${ }^{1,2}$ and \\ Fushan $\mathrm{Li}^{1,2^{*}}$
}

\begin{abstract}
Recently, solution-processed quantum dot lightemitting diodes (QLEDs) have emerged as a promising candidate for next-generation lighting and display devices. However, when given a constant voltage or current, the QLEDs need a certain working time to reach their maximum brightness. Such positive aging challenge, dramatically reducing the response speed of the device and causing a luminescence delay, is urgent to be investigated and resolved. In the current work, we introduce a charge-storage layer architecture by inserting copper(I) thiocyanate (CuSCN) between the organic holeinjection layer and hole-transport layer. The extracted holes will be released during the next electrical signal stimulation to increase the efficiency of charge transport. As a result, the response speed of the QLEDs is improved by an order of magnitude. In addition, by inserting an inorganic $\mathrm{CuSCN}$ layer, the efficiency, lifetime, and environmental stability of red/green/blue full-color QLEDs are enhanced simultaneously. Moreover, this work provides a generic strategy for the fabrication of fast-response and high-efficiency full-color QLEDs without luminescence delay, which plays a critical role in the practical industrialization of QLEDs.
\end{abstract}

Keywords: quantum dot, response speed, luminescence delay, charge storage layer

\section{INTRODUCTION}

Colloidal quantum dot light-emitting diodes (QLEDs) have attracted considerable attention in the display and lighting fields due to their unique advantages, such as high color purity, tunable emission wavelength covering the entire visible region as well as the low manufacturing cost [1-9]. To the present, QLEDs have achieved enormous progress in device efficiency by improving quantum yields (QYs) of quantum dots (QDs), balancing device charge carriers, and restricting carrier quenching [10-18]. According to the recent results, the efficiency of QLEDs has been comparable to that of organic light emitting diodes (OLEDs) [3,19-21]. However, the previous studies have demonstrated that QLEDs require a long aging process to achieve the stable light emission [22,23], a luminescence delay phenomenon that the brightness (or efficiency) of QLEDs increases progressively over time. This process can last for several days or even weeks, until the luminous efficiency reaches its maximum. When the device is continuously maintained in an energized state or is heated, this process will be shortened to minutes or even seconds. This is called the forward aging of the QLEDs, of which the physical mechanism remains unclarified $[24,25]$. As a result, this phenomenon will lead to a delay in the light emission of the QLEDs, and severely limit the application of QLEDs in the field of high-dynamic display. However, there is still no clear solution to accelerate the positive aging process in order to enhance the response speed and stability of the device [26-28].

The present work proposes a novel poly(3,4-ethylenedioxythiophene):poly(4-styrenesulfonate) (PEDOT:PSS)/copper(I) thiocyanate (CuSCN) charge-storage layer (CSL) structure, which can address the above-mentioned challenges, achieving fast-response and high-efficiency QLEDs. By inserting the inorganic hole-storage layer CuSCN between PEDOT:PSS and the hole-transport layer (HTL), a portion of the holes could be captured in the CSL during voltage stimulation and be retained for a period of time even after the removal of electrical stimulation. To increase the efficiency of charge transport, the trapped holes will be released during the next electrical signal stimulation. This special charge collection capability is extremely conducive to solving the luminescence delay in QLEDs. By adjusting the capture and release of charges in the CSL, the response speed of the device is increased by an order of magnitude. In addition, based on a deep valence-band maximum (VBM) of $-5.5 \mathrm{eV}$, CuSCN could effectively alleviate the energy barriers between the PEDOT:PSS and HTL, which is of great benefits for hole injection. In addition, the external quantum efficiency (EQE) and lifetime are improved by approximately $40 \%$ and $100 \%$, respectively, which are higher than those of control devices without CuSCN layer. The moisture absorbed by the PEDOT: PSS layer can be blocked from penetrating into the HTL, which can thus enhance the environmental stability of devices. The lifetime of the CSL device is over 100 times longer in comparison with that of control devices. The results demonstrate that the strategy based on organic/inorganic PEDOT:PSS/CuSCN CSL structure exhibits promising potential to achieve fastresponse QLEDs with high efficiency and stability.

\footnotetext{
${ }^{1}$ Institute of Optoelectronic Technology, Fuzhou University, Fuzhou 350116, China

${ }^{2}$ Fujian Science \& Technology Innovation Laboratory for Optoelectronic Information of China, Fuzhou 350116, China

These authors contributed equally to this work.

* Corresponding author (email: fsli@fzu.edu.cn)
} 


\section{EXPERIMENTAL SECTION}

\section{Materials preparation}

Unless otherwise specified, all reagents were purchased from commercial sources and used directly. The CuSCN (99\%, from Sigma-Aldrich) powder was dissolved in diethyl sulfide (DES, $98 \%$, Sigma-Aldrich) at a concentration of $30 \mathrm{mg} \mathrm{mL}^{-1}$ by stirring at $60^{\circ} \mathrm{C}$ for $12 \mathrm{~h}$. In addition, PEDOT:PSS and poly[(9,9dioctylfluorenyl-2,7-diyl)-co-(4,4'-(N-(4-sec-butyl-phenyl) diphenylamine)] (TFB) were purchased from Xi'an Polymer Light Technology Corp. TFB was dissolved in chlorobenzene at a concentration of $8 \mathrm{mg} \mathrm{mL}^{-1}$, which was heated and stirred at $60^{\circ} \mathrm{C}$ for $1 \mathrm{~h}$. The red, green, and blue CdSe/ZnS QDs were dispersed in $n$-octane at concentrations of $15,12.5$, and $10 \mathrm{mg} \mathrm{mL}^{-1}$, respectively. Then, $\mathrm{ZnO}$ nanoparticles (NPs) were synthesized according to the previously reported procedure with some modifications [29]. Zinc acetate dehydrate $(0.59 \mathrm{~g}, 99 \%$ purity, from Sigma-Aldrich) was dissolved in $25 \mathrm{~mL}$ of methanol and heated by water-bath at $63^{\circ} \mathrm{C}$. Subsequently, $0.3 \mathrm{~g} \mathrm{KOH}$ $(4.6 \mathrm{mmol})$ was dissolved in $13 \mathrm{~mL}$ of methanol. Meanwhile, the $\mathrm{KOH}$ solution was added dropwise to the zinc acetate solution at a uniform rate. Then, the solution was stirred for $2.5 \mathrm{~h}$ in a water bath at $63^{\circ} \mathrm{C}$. The precipitated $\mathrm{ZnO}$ NPs were centrifugated and washed with methanol for twice, and then redispersed in $13 \mathrm{~mL}$ of $n$-butanol solution. A given amount of polymer polyvinylpyrrolidone (PVP) was dissolved in the $\mathrm{ZnO} \mathrm{NP}$ solution at room temperature while keeping stirring for nearly $30 \mathrm{~min}$. All materials were filtered through the filter before use.

\section{Device fabrication}

Acetone, ethanol and deionized water were used to ultrasonically clean the patterned indium tin oxide (ITO) glass substrate for $15 \mathrm{~min}$, which was blow-dried with nitrogen before use. The surface of the ITO glass was treated by $\mathrm{O}_{2}$ plasma for $10 \mathrm{~min}$ in order to enhance the wettability for spin coating. PEDOT:PSS and CuSCN films were spin-coated on ITO in sequence at $3000 \mathrm{rmin}^{-1}$ for $40 \mathrm{~s}$ and dried at $120^{\circ} \mathrm{C}$ for $20 \mathrm{~min}$. Subsequently, the sample was transferred into a nitrogen-filled glove box to deposit other function layers. The rotational speed of the TFB functional layer was $3000 \mathrm{r} \mathrm{min}^{-1}$. After $40 \mathrm{~s}$ of spin coating, it was heated at $120^{\circ} \mathrm{C}$ for $10 \mathrm{~min}$. The rotation speeds of the QD layer and the $\mathrm{ZnO}$ layer were set to $2000 \mathrm{r} \mathrm{min}^{-1}$. After $40 \mathrm{~s}$ of spin coating, the sample was heated at $80^{\circ} \mathrm{C}$ for $10 \mathrm{~min}$. Finally, $100 \mathrm{~nm}$ Ag electrode was deposited by thermal evaporation through a shadow mask under a vacuum of $\sim 8 \times 10^{-4}$ Torr $(1$ Torr $=133.3 \mathrm{~Pa})$. Besides, the overlapping area of the patterned ITO on the bottom and the Ag electrode deposited on the top determines the effective light-emitting area of the device $\left(4 \mathrm{~mm}^{2}\right)$.

\section{Characterization}

The current-voltage $(I-V)$ characteristics were measured with a semiconductor characterization system (Keithley 4200-SCS). A silicon photodiode was employed to measure the brightness of devices. A fiber optic spectrometer (Ocean Optics USB2000+) was used to measure the electroluminescence (EL) spectra of QLEDs. Atomic force microscope (AFM, Bruker, Multimode 8) was adopted to detect the surface morphology of the film. Transmission electron microscope (TEM, TECNAL G2 F20) was applied to characterize the microscopic morphology of QDs. Additionally, time-resolved photoluminescence (TRPL) spec- trometer was used to measure the fluorescence lifetime of QDs. The device lifetime tests were performed with an aging lifespan test instrument.

\section{RESULTS AND DISCUSSION}

In recent years, it has been reported that the efficiency and brightness of QLEDs will significantly increase after being placed for a period of time [22-25], which is also known as the positive aging of QLEDs. Moreover, this process will be greatly accelerated by the continuous energization or heating of the devices. Given a constant voltage, the device needs a certain working time to reach its maximum brightness (i.e., luminescence delay). Fig. 1a illustrates a standard QLED with a architecture of ITO/ PEDOT:PSS/TFB/QDs/ZnO/Ag. The corresponding energy level diagram is shown in Fig. 1b. The normalized EL spectra of the QLEDs are illustrated in Fig. 1c. The symmetric emission peaks of blue (B), green (G) and red (R) QLEDs locate at 466, 530 and $635 \mathrm{~nm}$ with a narrow full-width at half-maximum of 25, 21 and $26 \mathrm{~nm}$, respectively (ultraviolet-visible absorption and normalized PL spectra of QDs, more details can be found in Figs S1-S3). Regarding these QLEDs, we also observed the positive aging behavior. At the same time, a constant voltage of $5 \mathrm{~V}$ was applied to the device to accelerate the process. As confirmed in Fig. 1d, the current and brightness of the PEDOT: PSS-based device gradually increase under the bias voltage, and it will take a certain time (about $3 \mathrm{~s}$ ) to reach a relatively stable state. After removing the stimulus voltage, the device current was re-tested after different time intervals (the waveform of the applied pulse displayed in Fig. S4). The device current reincreases from the initial state to the saturated one. The inset proves the luminescence delay of the device under constant voltage stimulations. Video S1 provides more specific details. In addition, we applied pulse voltages $(5 \mathrm{~V}, 100 \mathrm{~ms}, 0.5 \mathrm{~Hz})$ to the device and changed the interval between two pulse voltages. With increasing interval time, the luminescence delay of the device becomes more obvious. In order to further investigate the origin of the delay, a hole-only device structure of ITO/PEDOT: $\mathrm{PSS} / \mathrm{TFB} / \mathrm{Au}$ was prepared. The red curve in Fig. 1e represents the device responses under seven consecutive dual positive voltage sweeps $(0 \rightarrow 1.5 \rightarrow 0 \mathrm{~V})$. The current of the device continuously increases and becomes saturated when the number of voltage sweeps increases. By repeating the above seven consecutive dual positive voltage sweeps $(0 \rightarrow 1.5 \rightarrow 0 \mathrm{~V})$, the measured device current (black curve) increases from the initial state to the saturated state again. Furthermore, we employed 30 identical pulse voltages $(5 \mathrm{~V}, 100 \mathrm{~ms}, 0.5 \mathrm{~Hz})$ to the device in order to measure the current change of the device (Fig. 1f). As the number of pulses increases, the current of the device continues to increase and becomes saturated. Similarly, regardless of whether it is retested after a short time interval of 1 or $10 \mathrm{~s}$, the current of the PEDOT:PSS-based device increases from the initial state to the saturated state again. Based on these results, it is possible to remove the luminescence delay phenomenon of the device via the HTL engineering.

It is well known that the electron injection capacity in QLEDs is higher than that of holes. Large energy gap between the HTL and emissive layer (EML) forms a significant barrier that would hinder the hole injection into the QDs. Improving the hole injection efficiency may provide an effective way to solve the problem of luminescence delay of the device. In the present study, a CSL is introduced into the QLEDs device. The sche- 


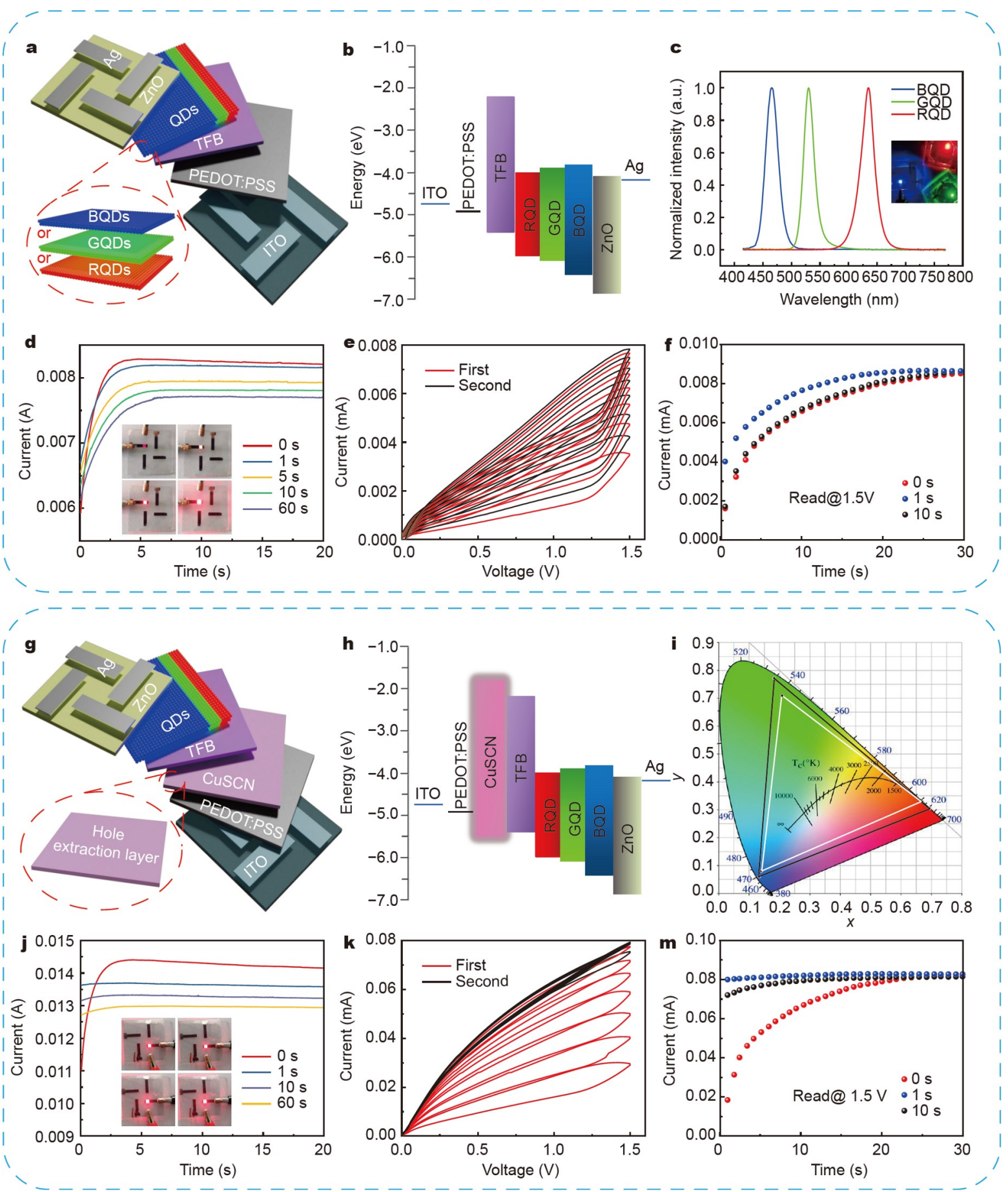

Figure 1 Device performance and luminescence delay test of full-color QLEDs. (a) Standard device structure. (b) Energy band diagram of the QLEDs. (c) The normalized EL spectra. (d) Luminescence delay test chart under constant voltage and the illustration shows the photo of the device's luminescence delay. (e) $I$ - $V$ curves of single-hole devices in two different time periods under seven consecutive dual positive voltage sweeps. (f) Current change curve under pulse voltage scanning. (g) The schematic device structure of the CSL QLEDs. (h) Energy band diagram. (i) CIE chromaticity diagram. (j) The test chart for eliminating the device's luminescence delay at a constant voltage and the illustration presents a photo of the device without luminescence delay. (k) $I$ - $V$ curves of CSL single-hole devices in two different time periods under seven consecutive dual positive voltage sweeps. $(\mathrm{m})$ The current curve of the CSL device under pulse voltage scanning.

matic device structure is shown in Fig. 1g, which consists of ITO/PEDOT:PSS/CuSCN/TFB/QDs/ZnO/Ag. As a p-type semiconductor, the $\mathrm{CuSCN}$ layer has unique hole extraction properties [29]. This special charge collection capability is extremely beneficial for eliminating the luminescence delay phenomenon (the specific reasons will be explained in detail later). All devices show saturated and pure colors as demonstrated by the Commission International de l'Eclairage (CIE) chromaticity diagram in Fig. 1i. The National Television System Committee standard color triangle is completely covered by the 
color gamut of our full-color QLEDs with 20\% improvement. To observe the luminescence delay characteristics, we also applied a constant voltage of $5 \mathrm{~V}$ to the CSL-based device, as shown in Fig. 1j. During the first round voltage simulation, the device current gradually increases and saturates within $2 \mathrm{~s}$. However, after removing the stimulus voltage, we re-tested the device current after different time intervals (the waveform of the applied pulse is denoted in Fig. S4). Starting from the second round voltage stimulation, the current and brightness of the CSL-based device reach a stable value rapidly (about $0.1 \mathrm{~s}$ ), and the response speed has increased by an order of magnitude. The inset presents a photo of the device without luminescence delay under constant voltage stimulation (for more specific details, please refer to Video S2). Furthermore, a hole-only device was also fabricated with ITO/PEDOT:PSS/CuSCN/TFB/Au structure. The red curve in Fig. 1k indicates the device current under the first seven consecutive dual positive voltage sweeps $(0 \rightarrow 1.5 \rightarrow 0 \mathrm{~V})$. After a cycle of the first seven consecutive dual positive voltage sweeps $(0 \rightarrow 1.5 \rightarrow 0 \mathrm{~V})$, the same second cycle is then repeated. Obviously, the black curve current measured by repeated scanning has stabilized in a saturated state thereafter. Similarly, we applied 30 identical pulse voltages $(5 \mathrm{~V}, 100 \mathrm{~ms}$, $0.5 \mathrm{~Hz}$ ) to the device (Fig. $1 \mathrm{~m}$ ). Interestingly, regardless of whether it is retested after a time interval of 1 or $10 \mathrm{~s}$, the current of the CSL-based device remains in a saturated state. Clearly, due to the insertion of the CSL, the phenomenon of luminescence delay is significantly alleviated.

In order to investigate the origin of the luminescence delay for the QLEDs, we tested the conductance changes of PEDOT:PSSbased and CSL-based devices during voltage application (see Fig. S5 for more details). As illustrated in Fig. 2a, with the first application of voltage, the conductance of the PEDOT:PSS-based device gradually switches to a high conductance state. This may be attributed to hole trap in the defects level at the PEDOT:PSS/ TFB interface. Nevertheless, after removing the voltage stimulus, the conductance quickly returns to the initial state due to the release of holes from the shallow trap levels. During the second voltage stimulation, the conductance of the device also increases slowly from the initial state to the high conductance state. The CSL improves the respond speed of the QLEDs, which is achieved by ingeniously integrating a CuSCN/TFB memristor interface into our device. Regarding the CSL-based device, as shown in Fig. 2b, with the first application of voltage, the conductance of the device also progressively becomes a high conductance state. However, the conductance will not return to the initial low conductance state after the removal of the voltage stimulation. The CuSCN layer, as a p-type semiconductor, possesses unique hole-collecting properties. The charge could be captured in the CSL during voltage stimulation and be retained for a period of time even after the electrical stimulation is removed. Furthermore, we measured the capacitance-voltage characteristics of the CSL and the control device, and verified that the introduction of $\mathrm{CuSCN}$ could indeed trap the charges (see Fig. S6 for more details). The TFB film exhibited a strong PL emission centered at $430 \mathrm{~nm}$ (Fig. 2c). In the presence of the CuSCN charge-extraction layer, the PL emission of the TFB film is strongly quenched, from which transfer of holes across the interfaces could also be inferred [30,31]. Furthermore, we tested the absorption spectra of CuSCN and PEDOT:PSS in the TFB emission range. The normalized PL spectra of the TFB are shown in Fig. S7. According to the results, the absorption of
CuSCN is extremely limited in the TFB emission range. It is proved that the lower PL intensity of CuSCN/TFB is not due to the absorption of CuSCN. To account for the whole process more clearly, we further analyzed the charge transfer process in QLEDs during electrical signal stimulation. As shown in Fig. 2d, e, in comparison with the control devices, the CuSCN layer can capture a portion of the holes during the first voltage stimulation. After the removal of electrical stimulation, the holes can be maintained for a period of time. The trapped holes are released during the next electrical signal stimulation to increase the efficiency of charge transfer at the CuSCN/TFB interface. The trapped holes in CSL can moderate the energy barrier between the HTL and QD EML, and promote the hole injection and transportation.

Fig. S8 shows the X-ray diffraction (XRD) pattern of the CuSCN thin film deposited on the TFB layer. The distinct peaks are in consistence with the typical diffractions of (003), (101) and (012) planes, revealing the formation of rhombohedral phase ( $\beta$-phase) of the CuSCN film, which is a wide-band-gap semiconductor [32]. Furthermore, to confirm the feasibility of CSL for QLEDs, it is of necessity to control its film quality because the interface of multilayer films in QLEDs exerts a critical impact on the device performance. Fig. S9a-c present the AFM topographic images for PEDOT:PSS, CuSCN and PEDOT: PSS/CuSCN deposited on the ITO glass substrate, respectively. When deposited directly on ITO (Fig. S9b), the surface of the CuSCN film is embossed with raised particles, with a root-meansquare surface roughness (RMS) of $2.9 \mathrm{~nm}$. Apparently, uniform CuSCN film is attained on the surface of PEDOT:PSS, with an RMS of $2.8 \mathrm{~nm}$, thus providing the possibility to deposit highquality carrier transport and light-emitting layers. Fig. S10a-c present the high-resolution TEM (HRTEM) images of the red, green, and blue (R, G, and B) QDs. It can be seen from Fig. S10d-f that the QDs exhibit uniform size distribution. By adjusting the size of the QDs, their color can be controlled and the size of red QDs $(11.7 \mathrm{~nm})$ is slightly larger in comparison with that of green $(8.9 \mathrm{~nm})$ and blue $(7.8 \mathrm{~nm})$ QDs. HRTEM image in the illustration shows a clear QD lattice structure and the obvious lattice fringes confirm the high crystallinity of QDs. As presented in Fig. S10g-i, the prepared full-color QD films show excellent flatness, which is beneficial for the fabrication of high-performance QLEDs.

Another important advantage of CuSCN refers to its capability of efficiently injecting holes in QLEDs due to its deeper hole injection level. The energy band diagram of the CSL device is shown in Fig. 1b. After coating the CuSCN layer, the valence band level of hole injection layer (HIL) is shifted down to $-5.5 \mathrm{eV}$, which is prospectively beneficial for hole injection into the QDs EML. The energy offset between the HIL and HTL is reduced. Therefore, the CSL has more suitable energy alignment with TFB to promote the charge transport. The current densityvoltage $(J-V)$, luminance-voltage $(L-V)$ and current efficiencyluminance (CE- $L$ ), luminance-EQE ( $L$-EQE) characteristics of full-color devices are recorded in Fig. 3a-f (see Fig. S11 for more details). Meanwhile, the corresponding performance of control devices is also presented as a reference. For all (R, G and $B)$ devices, the current densities of CSL devices are lower than those of the control devices under $6 \mathrm{~V}$, and the difference in current density increases with voltage (the EL spectrum evolution under different applied voltages for the CSL-based green QLEDs is displayed in Fig. S12). Moreover, the maximum EQE is 

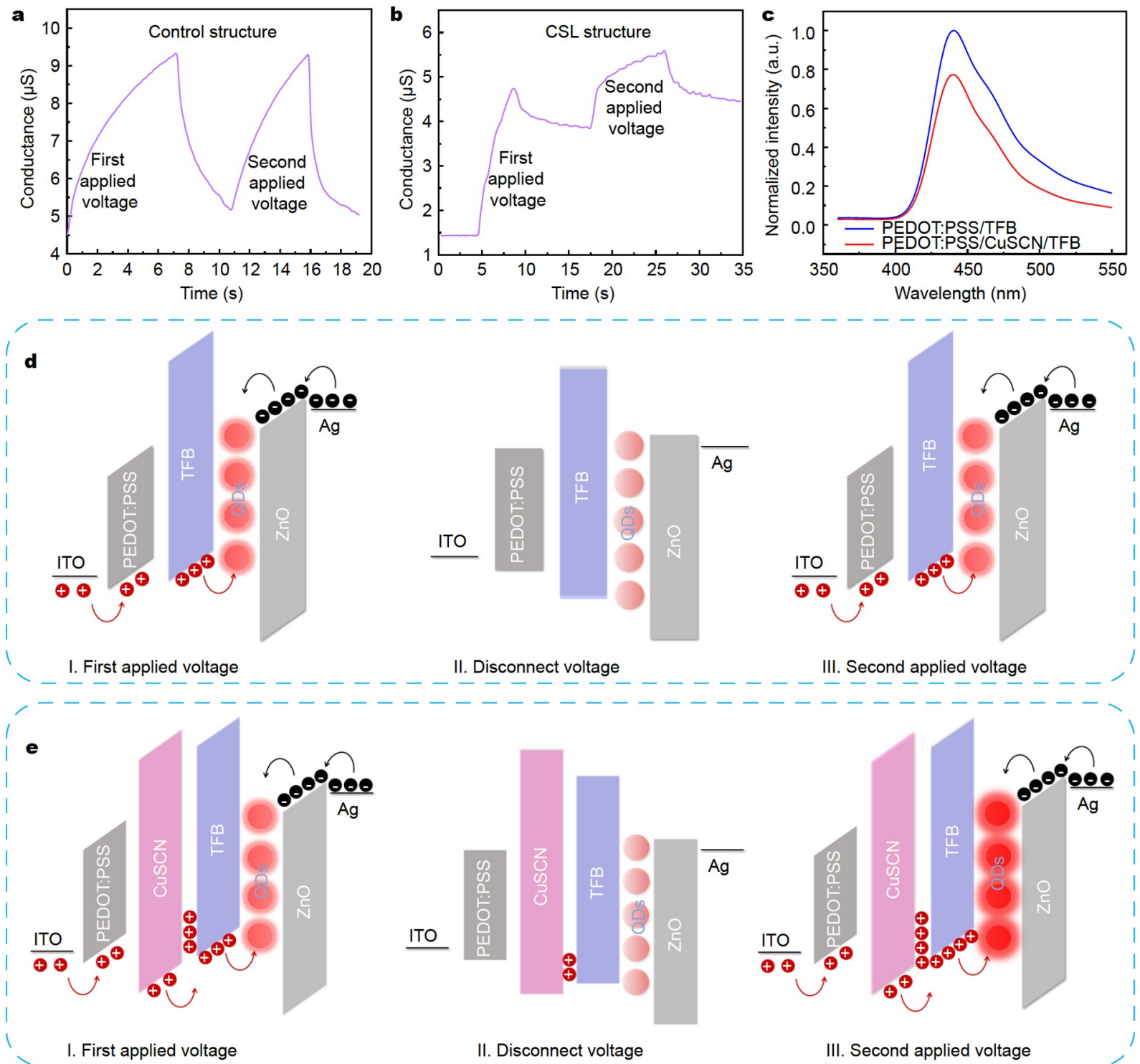

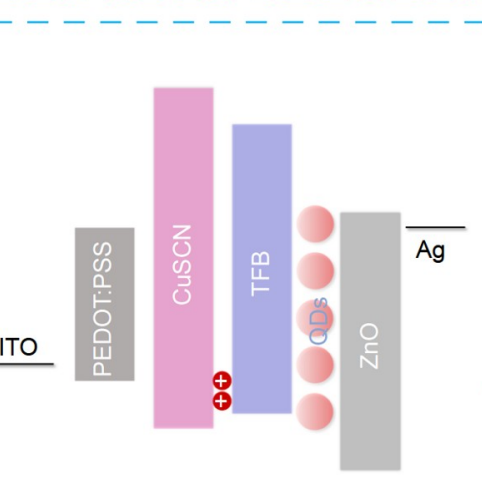

II. Disconnect voltage

III. Second applied voltage

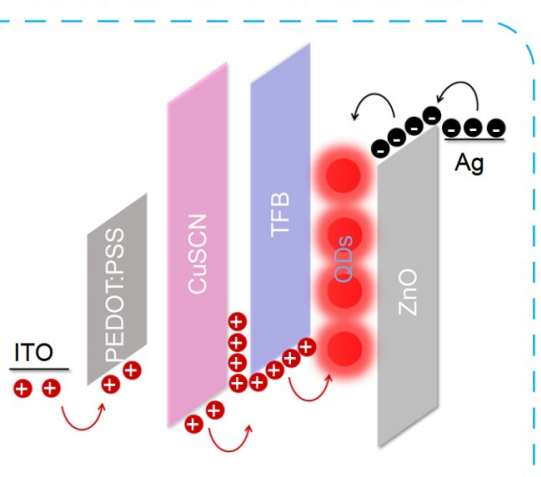

III. Second applied voltage

Figure 2 Luminescence delay mechanism. Change in conductance of the (a) control and (b) CSL structures during voltage application. (c) Steady-state PL. Schematic diagram of the charge transfer process of a QLED with the (d) control and (e) CSL structures during electrical signal stimulation.

obviously enhanced from $11.2 \%(\mathrm{R}), 8.2 \%(\mathrm{G})$ and $4.7 \%(\mathrm{~B})$ for control devices to $15.4 \%(\mathrm{R}), 11.2 \%(\mathrm{G})$ and $6.5 \%(\mathrm{~B})$ for CSL devices. The device performance is summarized in Table 1.

Fig. $3 g$ shows that the CuSCN film has extremely high transparency, and the insertion of the CuSCN layer maintains the high transmittance of the charge-transport layers. In addition, with green QDs (GQDs) as a representative, we prepared the CSL-based and PEDOT:PSS-based hole-only devices (ITO/ $\mathrm{HIL} / \mathrm{TFB} / \mathrm{GQDs} / \mathrm{Au}$ ). Fig. $3 \mathrm{~h}$ provides the $J-V$ characteristics of the above hole-only devices, demonstrating that the current density of the CSL-based hole-only device is almost one order of magnitude greater than that of the PEDOT:PSS-based one. This is consistent with our previous analysis that CuSCN can effectively increase the hole injection capability of the device. In addition, we also tested the durability of the hole-only devices under the same environmental conditions. Fig. $3 \mathrm{~h}$ shows that the CSL-based device exhibits better environmental stability in comparison with the PEDOT:PSS-based device. Furthermore, the fluorescence lifetime curves of QD films by TRPL spectroscopy are illustrated in Fig. 3i. The TEM images of $\mathrm{ZnO}$ and $\mathrm{ZnO}: \mathrm{PVP}$ composite at different resolutions are displayed in Figs S13 and S14.

The CSL-based QLEDs exhibit outstanding lifetime even under high-brightness conditions. As confirmed in Fig. $4 a-c$, the devices were tested at a constant driving current of $5 \mathrm{~mA}$, conforming to different initial luminances $\left(L_{0}\right)$. The half-lifetime, $T_{50}$, is defined as the time for the luminance decreased to $L_{0} / 2$. Based on the relation $L_{0}{ }^{n} T_{50}=$ constant, and assuming an acceleration factor of $n=1.7$, the lifetime of CSL-based RGB devices is more than twice that of PEDOT:PSS-based devices. This may be also attributed to the efficient hole injection capability of CuSCN, significantly alleviating the hole aggregation at the HTL/QDs interface and the consequent joule heating effect. In addition, by inserting the CuSCN intermediate layer, the moisture absorbed by the PEDOT:PSS layer can be blocked from penetrating into the TFB layer, generating obviously enhanced 


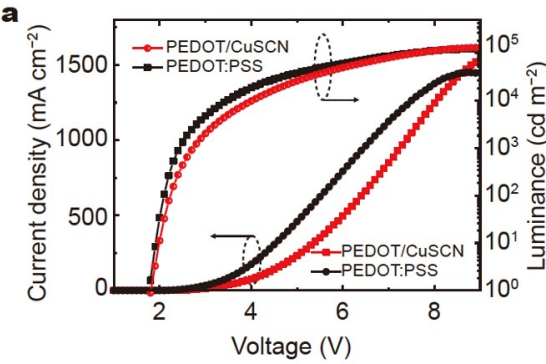

d

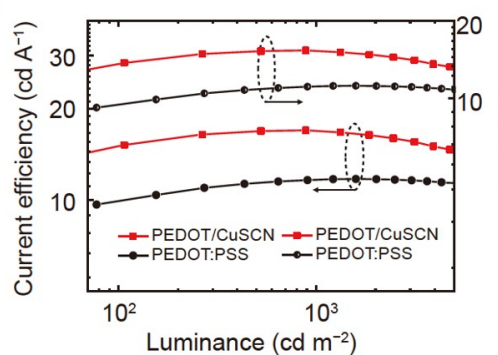

g

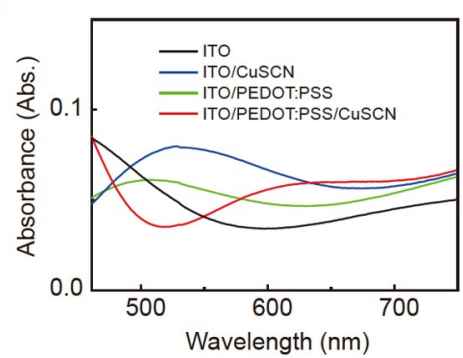

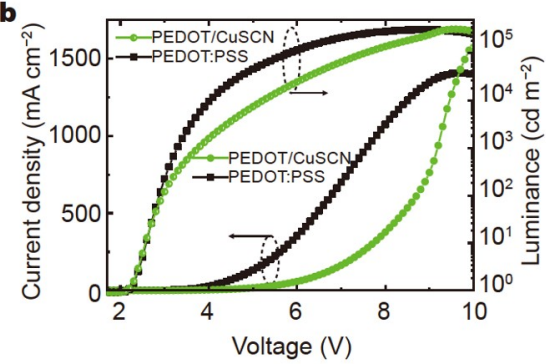
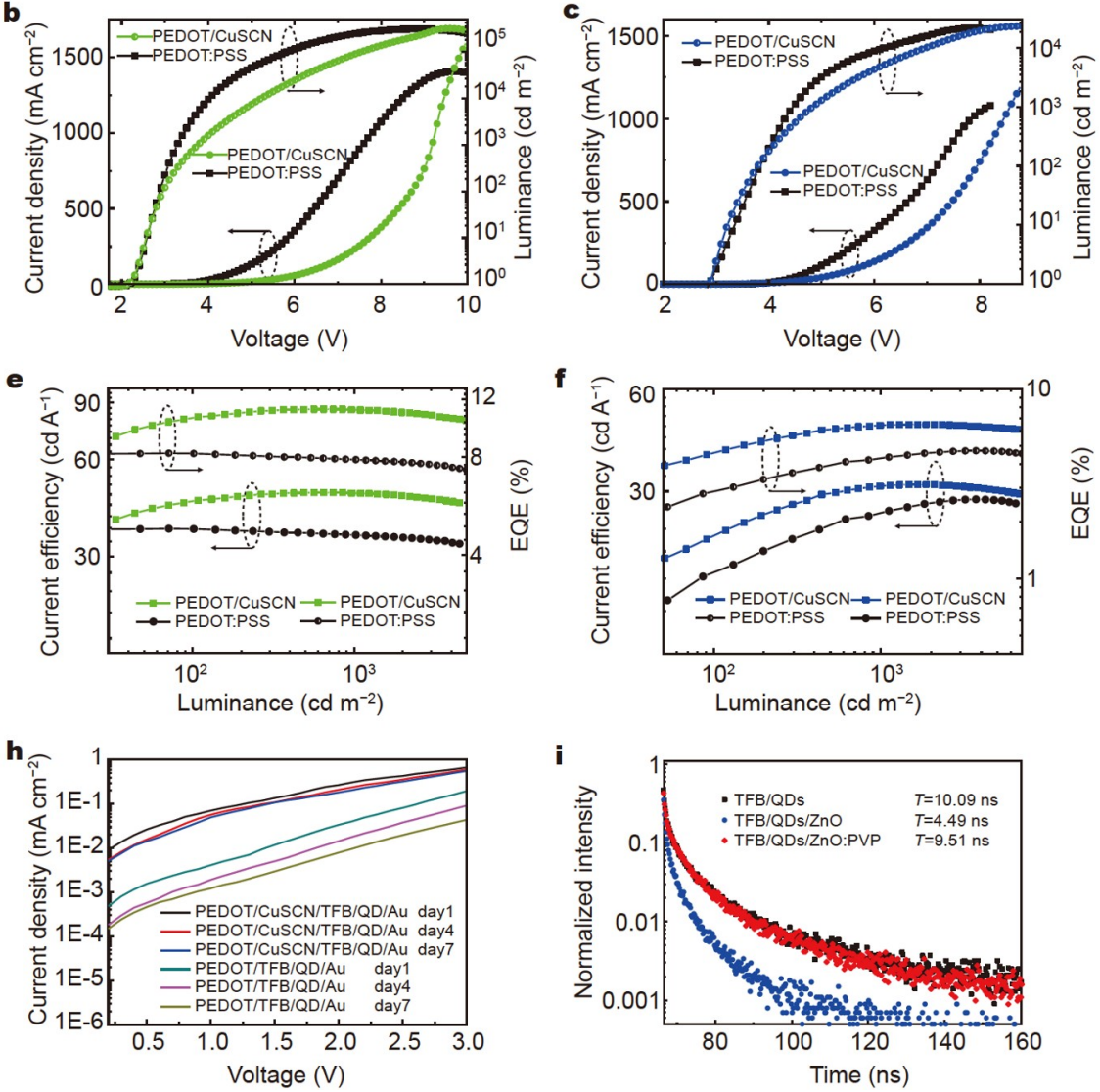

i

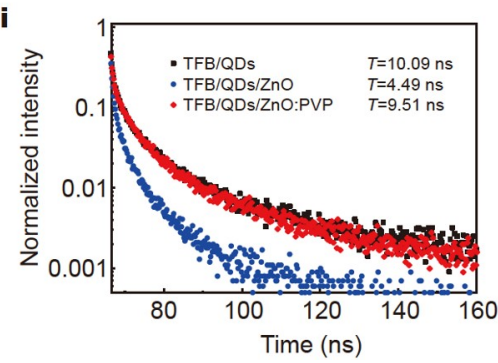

Figure 3 EL performance measurement and characterization. $J-V, V$ - $L$ characteristics of the (a) red, (b) green and (c) blue devices. CE- $L$, $L$-EQE characteristics of the (d) red, (e) green and (f) blue devices. (g) Absorption spectrum of each functional layer films on top of ITO. (h) Comparison of $J$ - $V$ characteristics of both hole-only devices. (i) TRPL decay for the QD films.

Table 1 Summary of the main performance parameters of full-color device

\begin{tabular}{|c|c|c|c|c|c|c|}
\hline \multirow{2}{*}{ Type of device } & \multirow{2}{*}{ Turn-on voltage (V) } & \multirow{2}{*}{$L_{\max }\left(\mathrm{cd} \mathrm{m}^{-2}\right)$} & \multicolumn{2}{|c|}{$\eta_{\mathrm{A}}\left(\mathrm{cd} \mathrm{A}^{-1}\right)$} & \multicolumn{2}{|c|}{$\eta_{\mathrm{EQE}}(\%)$} \\
\hline & & & $1000 \mathrm{~cd} \mathrm{~m}^{-2}$ & Peak & $1000 \mathrm{~cd} \mathrm{~m}^{-2}$ & Peak \\
\hline $\begin{array}{c}\mathrm{PEDOT} / \mathrm{CuSCN} / / \mathrm{TFB} / \\
\mathrm{RQD} / \mathrm{ZnO}\end{array}$ & 1.9 & 130,000 & 16.7 & 16.9 & 15.2 & 15.4 \\
\hline $\begin{array}{c}\text { PEDOT:PSS/TFB/RQD/ } \\
\text { ZnO }\end{array}$ & 1.9 & 122,000 & 11.6 & 11.7 & 11.1 & 11.2 \\
\hline $\begin{array}{c}\mathrm{PEDOT} / \mathrm{CuSCN} / \mathrm{TFB} / \\
\text { GQD/ZnO }\end{array}$ & 2.2 & 316,000 & 44.8 & 47.3 & 10.6 & 11.2 \\
\hline $\begin{array}{c}\text { PEDOT:PSS/TFB/GQD/ } \\
\text { ZnO }\end{array}$ & 2.2 & 310,000 & 36.4 & 36.5 & 8.1 & 8.2 \\
\hline $\begin{array}{c}\mathrm{PEDOT} / \mathrm{CuSCN} / \mathrm{TFB} / \\
\mathrm{BQD} / \mathrm{ZnO}\end{array}$ & 2.9 & 23,000 & 3.1 & 3.2 & 6.4 & 6.5 \\
\hline $\begin{array}{c}\text { PEDOT:PSS/TFB/BQD/ } \\
\text { ZnO }\end{array}$ & 2.9 & 21,000 & 2.5 & 2.8 & 4.2 & 4.7 \\
\hline
\end{tabular}

device environmental stability. After one week of placement under the same environmental conditions, the lifetime of the CSL-based devices exceeded 100 times that of PEDOT:PSS-based devices (Fig. 4d). The RGB device environmental stability performance is summarized in Table S1.

\section{CONCLUSIONS}

To conclude, we demonstrated the realization of fast-response, high-stability, and high-efficiency full-color QLEDs using the
PEDOT:PSS/CuSCN CSL. By adjusting the capture and release of charges in the CuSCN layer, the response speed of the device is increased by an order of magnitude. In the meanwhile, the efficiency of the devices based on CSL was increased by nearly $40 \%$, the device lifetime was enhanced by approximately $100 \%$, and the device showed excellent environmental stability. The improved performances can be attributed to the deep hole injection level of CuSCN, effectively promoting the hole injection efficiency and reducing the hole aggregation at the HTL/QD 

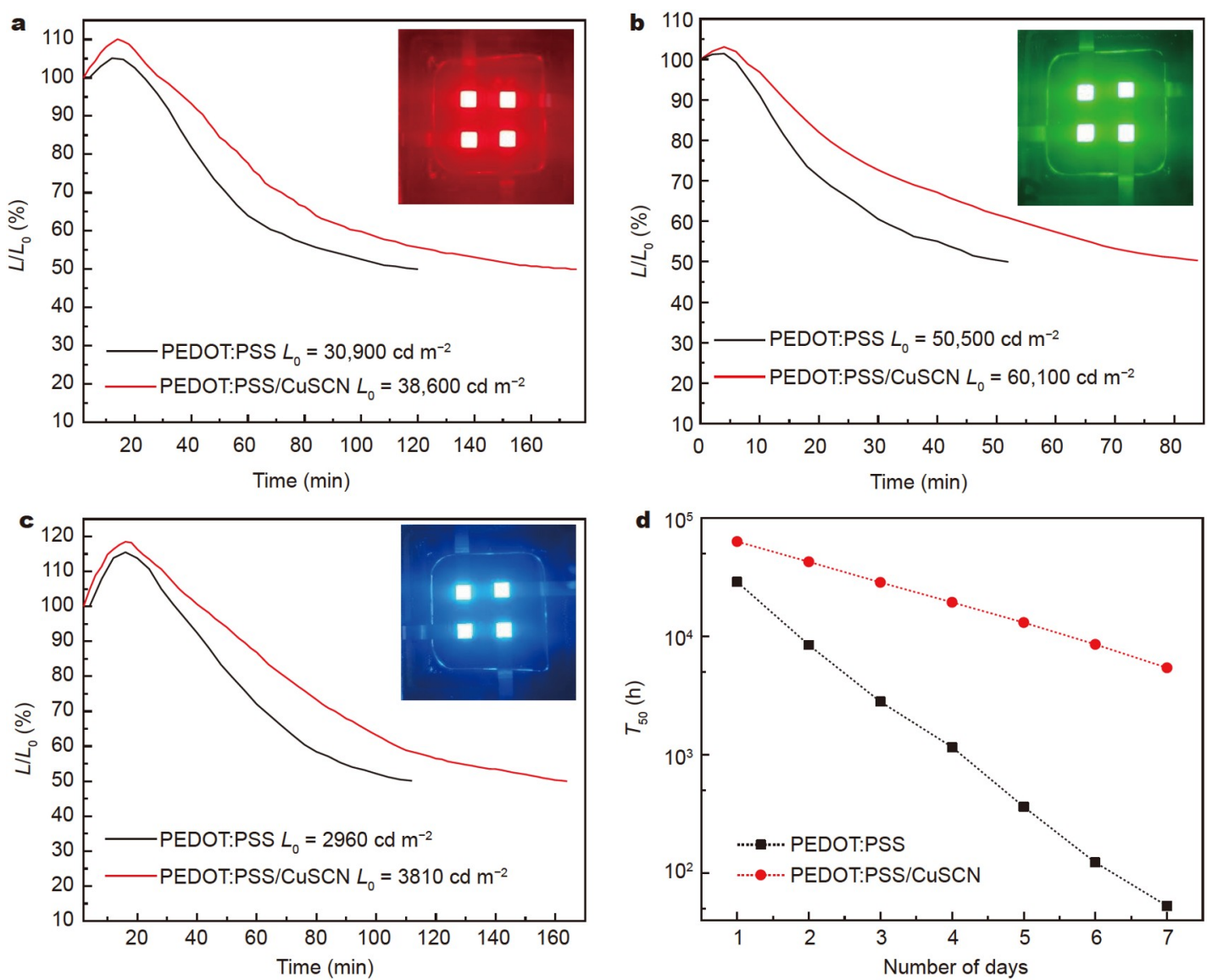

Figure 4 Lifetimes of full-color QLEDs. Lifetime comparison curves of (a) red, (b) green and (c) blue QLED devices with CSL structure and standard devices. (d) Environmental stability of green devices.

interface. In addition, the CuSCN layer could block the moisture from penetrating into the TFB layer to enhance the device environmental stability. Moreover, our work demonstrates the promising potential of QLEDs for application in the field of high-dynamic display by employing PEDOT:PSS/CuSCN CSL architecture.

\section{Received 11 August 2021; accepted 14 September 2021;} published online 29 October 2021

1 Dai X, Zhang Z, Jin Y, et al. Solution-processed, high-performance light-emitting diodes based on quantum dots. Nature, 2014, 515: 96-99

2 Mashford BS, Stevenson M, Popovic Z, et al. High-efficiency quantumdot light-emitting devices with enhanced charge injection. Nat Photon, 2013, 7: 407-412

3 Lin K, Xing J, Quan LN, et al. Perovskite light-emitting diodes with external quantum efficiency exceeding 20 percent. Nature, 2018, 562: 245-248

4 Zhang H, Su Q, Chen S. Quantum-dot and organic hybrid tandem light-emitting diodes with multi-functionality of full-color-tunability and white-light-emission. Nat Commun, 2020, 11: 2826

5 Oh N, Kim BH, Cho SY, et al. Double-heterojunction nanorod lightresponsive LEDs for display applications. Science, 2017, 355: 616-619

6 Qian L, Zheng Y, Xue J, et al. Stable and efficient quantum-dot lightemitting diodes based on solution-processed multilayer structures. Nat Photon, 2011, 5: 543-548

7 Li JS, Tang Y, Li ZT, et al. Toward 200 lumens per watt of quantum-dot white-light-emitting diodes by reducing reabsorption loss. ACS Nano, 2021, 15: 550-562
8 Su Q, Zhang $\mathrm{H}$, Chen S. Flexible and tandem quantum-dot lightemitting diodes with individually addressable red/green/blue emission. npj Flex Electron, 2021, 5: 8

9 Shen $\mathrm{H}$, Gao Q, Zhang Y, et al. Visible quantum dot light-emitting diodes with simultaneous high brightness and efficiency. Nat Photon, 2019, 13: 192-197

10 Xiang C, Wu L, Lu Z, et al. High efficiency and stability of ink-jet printed quantum dot light emitting diodes. Nat Commun, 2020, 11 1646

11 Dai X, Deng Y, Peng X, et al. Quantum-dot light-emitting diodes for large-area displays: Towards the dawn of commercialization. Adv Mater, 2017, 29: 1607022

12 Shen H, Cao W, Shewmon NT, et al. High-efficiency, low turn-on voltage blue-violet quantum-dot-based light-emitting diodes. Nano Lett, 2015, 15: 1211-1216

13 Wang $\mathrm{L}$, Lin $\mathrm{J}, \mathrm{Hu} \mathrm{Y}$, et al. Blue quantum dot light-emitting diodes with high electroluminescent efficiency. ACS Appl Mater Interfaces, 2017, 9: 38755-38760

14 Shi YL, Liang F, Hu Y, et al. High performance blue quantum dot lightemitting diodes employing polyethylenimine ethoxylated as the interfacial modifier. Nanoscale, 2017, 9: 14792-14797

15 Moon $\mathrm{H}$, Lee C, Lee W, et al. Stability of quantum dots, quantum dot films, and quantum dot light-emitting diodes for display applications. Adv Mater, 2019, 31: 1804294

16 Davidson-Hall T, Aziz H. Significant enhancement in quantum dot light-emitting device stability via a cascading hole transport layer. ACS Appl Mater Interfaces, 2020, 12: 16782-16791

17 Cao F, Wang H, Shen P, et al. High-efficiency and stable quantum dot light-emitting diodes enabled by a solution-processed metal-doped nickel oxide hole injection interfacial layer. Adv Funct Mater, 2017, 27: 


\section{8}

18 Cheng Y, Wan $\mathrm{H}$, Liang $\mathrm{T}$, et al. Continuously graded quantum dots: Synthesis, applications in quantum dot light-emitting diodes, and perspectives. J Phys Chem Lett, 2021, 12: 5967-5978

19 Liu D, Cao S, Wang S, et al. Highly stable red quantum dot lightemitting diodes with long $T_{95}$ operation lifetimes. J Phys Chem Lett, 2020, 11: 3111-3115

20 Zhang W, Ding S, Zhuang $\mathrm{W}$, et al. InP/ZnS/ZnS core/shell blue quantum dots for efficient light-emitting diodes. Adv Funct Mater, 2020, 30: 2005303

21 Joo WJ, Kyoung J, Esfandyarpour M, et al. Metasurface-driven OLED displays beyond 10,000 pixels per inch. Science, 2020, 370: 459-463

22 Su Q, Sun Y, Zhang H, et al. Origin of positive aging in quantum-dot light-emitting diodes. Adv Sci, 2018, 5: 1800549

23 Acharya KP, Titov A, Hyvonen J, et al. High efficiency quantum dot light emitting diodes from positive aging. Nanoscale, 2017, 9: 1445114457

24 Zhang W, Chen $\mathrm{X}$, Ma Y, et al. Positive aging effect of $\mathrm{ZnO}$ nanoparticles induced by surface stabilization. J Phys Chem Lett, 2020, 11: 5863-5870

25 Ding S, Wu Z, Qu X, et al. Impact of the resistive switching effects in $\mathrm{ZnMgO}$ electron transport layer on the aging characteristics of quantum dot light-emitting diodes. Appl Phys Lett, 2020, 117: 093501

26 Chen Z, Su Q, Qin Z, et al. Effect and mechanism of encapsulation on aging characteristics of quantum-dot light-emitting diodes. Nano Res, 2021, 14: 320-327

27 Xue X, Dong J, Wang S, et al. Degradation of quantum dot light emitting diodes, the case under a low driving level. J Mater Chem C, 2020, 8: 2014-2018

28 Chen D, Chen D, Dai X, et al. Shelf-stable quantum-dot light-emitting diodes with high operational performance. Adv Mater, 2020, 32: 2006178

29 Zhu Y, Xu R, Zhou Y, et al. Ultrahighly efficient white quantum dot light-emitting diodes operating at low voltage. Adv Opt Mater, 2020, 8: 2001479

30 Arora N, Dar MI, Hinderhofer A, et al. Perovskite solar cells with CuSCN hole extraction layers yield stabilized efficiencies greater than 20\%. Science, 2017, 358: 768-771

31 Stranks SD, Eperon GE, Grancini G, et al. Electron-hole diffusion lengths exceeding 1 micrometer in an organometal trihalide perovskite absorber. Science, 2013, 342: 341-344

32 Jung JW, Chueh CC, Jen AKY. High-performance semitransparent perovskite solar cells with $10 \%$ power conversion efficiency and $25 \%$ average visible transmittance based on transparent $\mathrm{CuSCN}$ as the holetransporting material. Adv Energy Mater, 2015, 5: 1500486

Acknowledgements This work was financially supported by the National Natural Science Foundation of China (62075043), and Fujian Science \& Technology Innovation Laboratory for Optoelectronic Information of China (2021ZZ126).

Author contributions Li F initiated and coordinated the research; Zhu Y and Liu Y designed the samples; Zhu Y performed the experiments; Liu Y performed the data analysis; Zhu Y, Liu Y and $\mathrm{Hu} H$ wrote the paper with support from Li F; Xu Z, Bai J, Yang $\mathrm{K}$ and Guo $\mathrm{T}$ participated in the discussion and analysis of the data. All authors contributed to the general discussion.

Conflict of interest The authors declare that they have no conflict of interest.

Supplementary information Experimental details and supporting data are available in the online version of the paper.

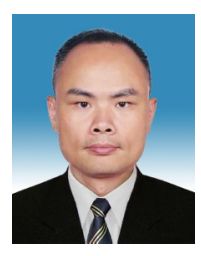

Fushan Li received his $\mathrm{PhD}$ degree from Peking University in 2005. He became a research professor at $\mathrm{Ha}-$ nyang University of Korea in 2007, and a research fellow at Nanyang Technological University in 2010. Now he is a full professor at Fuzhou University in China. His research mainly focuses on the nano-optoelectronic devices utilizing semiconductor nanoparticles, carbon-based nanomaterials and novel 2D atomic crystals.

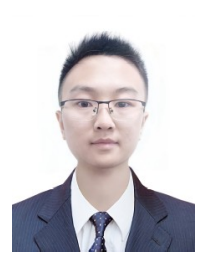

Yangbin Zhu is now a $\mathrm{PhD}$ student at the College of Physics and Information Engineering, Fuzhou University, under Prof. Fushan Li's supervision. His research topic is smart quantum dot light-emitting devices and artificial neural networks.

\section{具有电荷存储层的快速响应、高稳定、高效率全彩 量子点发光二极管}

朱阳斌 ${ }^{1,2 \dagger}$, 刘洋 ${ }^{1 \dagger}$, 胡海龙 ${ }^{1}$, 徐中炜 ${ }^{1}$, 白洁玉 ${ }^{1}$, 杨开宇 ${ }^{1}$, 郭太良 ${ }^{1,2}$, 李福山 ${ }^{1,2^{*}}$

摘要 溶液法处理的量子点发光二极管(QLEDs)已经成为下一代照明 和显示器件有希望的候选者. 然而, QLEDs的正向老化问题会极大地降 低器件的响应速度并导致发光延迟，严重限制了QLEDs器件在高动态 显示领域的应用. 本文中, 我们在有机空穴注入层和空穴传输层之间引 入电荷存储层硫凗酸亚铜 $(\mathrm{CuSCN})$, 存储的空穴将在下一次电信号刺 激期间释放以提高电荷传输效率，从而使QLEDs器件的响应速度提高 一个数量级. 此外, 通过插入无机CuSCN层, 红/绿/蓝全彩 QLEDs 器件 的效率、寿命和环境稳定性同时得到了提升. 这项工作为制造快速响 应和高性能的全彩QLEDs提供了一种通用策略. 